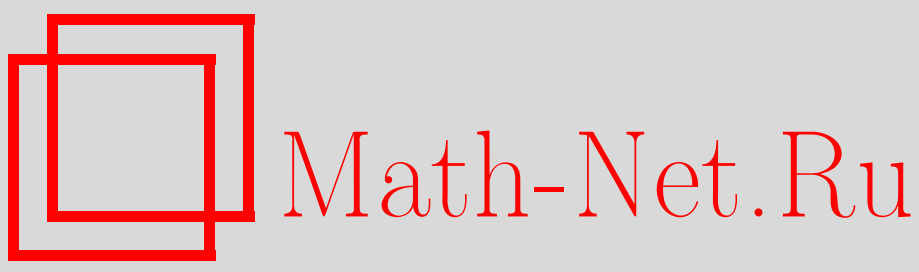

О. Г. Смолянов, С. А. Шкарин, Комплексная дифференцируемость по Гато и непрерывность, Изв. РАН. Сер. матем., 2004, том 68, выпуск 6, 157-168

DOI: https://doi.org/10.4213/im517

Использование Общероссийского математического портала Math-Net.Ru подразумевает, что вы прочитали и согласны с пользовательским соглашением http://www . mathnet.ru/rus/agreement

Параметры загрузки:

IP: 54.197 .130 .99

26 апреля 2023 г., $17: 51: 42$ 
УДК 517.98

\author{
О.Г. Смолянов, С.А. Шкарин
}

\title{
Комплексная дифференцируемость по Гато и непрерывность
}

\begin{abstract}
Известно, что на вещественных локально выпуклых пространствах $\mathcal{D}(\mathbb{R})$ финитных бесконечно дифференцируемых функций и $\mathcal{D}^{\prime}(\mathbb{R})$ обобщенных функций сушествуют всюду разрывные бесконечно дифференцируемые по Фреше функции. В работе рассматривается связь комплексной дифференцируемости функции на комплексном локально выпуклом пространстве с ее непрерывностью. Описан класс комплексных локально выпуклых пространств, включающий комплексное пространство $\mathcal{D}^{\prime}(\mathbb{R})$, такой, что всякая комплексно дифференцируемая по Гато функция на пространстве этого класса непрерывна. Описан другой класс локально выпуклых пространств, включающий комплексное пространство $\mathcal{D}(\mathbb{R})$, такой, что на каждом пространстве из этого класса существует всюду разрывная комплексно бесконечно дифференцируемая по Фреше функция, производные которой непрерывны.

Библиография: 21 наименование.
\end{abstract}

\section{§. Введение}

Следуюшие две теоремы доказаны в работе [6].

Теорема I. Пусть Е - вещественное топологическое векторное пространство. Тогда следующие два условия әквивалентны:

(i) любая дифферениируемая по Фреше в точке $x_{0} \in E$ функиия $f: E \rightarrow \mathbb{R}$ непрерывна в точке $x_{0}$;

(ii) Е является пространством Фреше-Урысона ${ }^{1}$.

Через $\mathcal{D F} \mathcal{S}$ будем обозначать класс всех локально выпуклых пространств (ЛВП), являющихся сильными сопряженньми к пространствам Фреше-Шварца [7], [8]. По-другому $\mathcal{D} \mathcal{F} \mathcal{S}$ можно описать как класс индуктивных пределов последовательностей банаховых пространств с компактными вложениями [7], [8]. Отметим, что бесконечномерные пространства из класса $\mathcal{D F} \mathcal{S}$ не бывают пространствами Фреше--Урысона.

Teорема II. Пусть $E \in \mathcal{D} \mathcal{F} \mathcal{S}, U \subseteq E$ - открытое множество и функиия $f: U \rightarrow \mathbb{R}$ всюду дифференцируема по Фреше. Тогда $f$ непрерьвна.

В то же время в работе [3] доказана следующая теорема.

Работа выполнена при поддержке РФФИ (грант № 02-01-01074).

${ }^{1}$ Топологическое пространство $X$ называется пространством Фреше-Урысона [9], если любая точка из замыкания множества $A \subset X$ является пределом последовательности элементов $A$. 
Tеорема III. Пусть Е - сепарабельное бесконечномерное вещественное хаусдорфово локально выпуклое пространство. Тогда существует всюду разрывная и всюду дифференцируемая по Гато функция $f: E \rightarrow \mathbb{R}$.

В работе [1] высказывается предположение, что на вещественном ЛВП $\mathcal{D}$ бесконечно дифференцируемых функций на $\mathbb{R}$ c компактным носителем существует вещественная функция, бесконечно дифференцируемая по Фреше и всюду разрывная. В статье [5] строится такая функция. В работе [4] строится функция на пространстве $\mathcal{D}^{\prime}$ обобшенных функций на $\mathbb{R}$ с теми же свойствами.

В настояшей работе решен вопрос о сушествовании аналогичных примеров в классе комплексно дифференцируемых функций на комплексных ЛВП $\mathcal{D}$ и $\mathcal{D}^{\prime}$. Более того, описан класс комплексных локально выпуклых пространств, включающий комплексное пространство $\mathcal{D}^{\prime}(\mathbb{R})$, такой, что всякая комплексно дифференцируемая по Гато функция на пространстве этого класса непрерывна. Описан другой класс локально выпуклых пространств, включающий комплексное пространство $\mathcal{D}(\mathbb{R})$, такой, что на каждом пространстве из этого класса существует всюду разрывная комплексно бесконечно дифференцируемая по Фреше функция, производные которой начиная с первой непрерьвны.

\section{§2. Обозначения и терминология}

Всюду ниже $\mathbb{K} \in\{\mathbb{R}, \mathbb{C}\}$. Пусть $E$ и $F-$ ЛВП над полем $\mathbb{K}$. Обозначим через $L(E, F)$ пространство линейных непрерывных отображений из $E$ в $F$, наделенное топологией равномерной сходимости [7], [8] на ограниченных подмножествах $E$. Вместо $L(E, \mathbb{K})$ мы пишем $E^{\prime}$.

Пусть $X$ и $Y$ - векторные пространства над полем $\mathbb{K}$. Через $\mathcal{B}^{n}(X, Y)$ будем обозначать пространство всех полилинейных отображений $\varphi: X^{n} \rightarrow Y$. Пространство всех симметричных полилинейных отображений из $\mathcal{B}^{n}(X, Y)$ обозначим $\mathcal{B}_{s}^{n}(X, Y)$. Для полунорм $p$ и $q$ на пространствах $X$ и $Y$ соответственно и отображения $\varphi \in \mathcal{B}^{n}(X, Y)$ положим

$$
\widehat{\varphi}(x)=\varphi(\underbrace{x, \ldots, x}_{n \text { раз }}), \quad p_{q}^{*}(\varphi)=\sup \left\{q\left(\varphi\left(x_{1}, \ldots, x_{n}\right)\right): x_{j} \in X, p\left(x_{j}\right) \leqslant 1\right\} .
$$

ОПРЕДЕЛЕНИЕ 1 [1], [6]. Пусть $E$ и $F$ - хаусдорфовы локально выпуклые пространства над полем $\mathbb{K}, U \subset E$ - открытое множество и $x_{0} \in U$. Отображение $f: U \rightarrow F$ называется дифференцируемым в точке $x_{0}$ по направлению $h \in E$, если существует предел

$$
D_{h} f\left(x_{0}\right)=\lim _{\substack{t \rightarrow 0 \\ t \in \mathbb{K}}} \frac{f\left(x_{0}+t h\right)-f\left(x_{0}\right)}{t} \in F .
$$

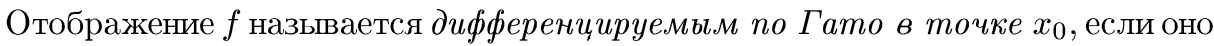
дифференцируемо в точке $x_{0}$ по направлению $h$ для всякого $h \in E$ и отображение $f^{\prime}\left(x_{0}\right): h \mapsto D_{h} f\left(x_{0}\right)$ является непрерывным линейным оператором.

Отображение $f$ называется дифференцируемым по Фреше в точке $x_{0}$, если $f$ дифференцируемо по Гато в $x_{0}$ и предел (2) равномерен по $h \in B$ для всякого ограниченного множества $B \subset E$. 
Если $f$ дифференцируемо по Гато во всех точках $U$, то отображение $f^{\prime}: U \rightarrow$ $L(E, F)$ корректно определено и можно рассматривать вопрос о дифференцируемости $f^{\prime}$. Мы считаем, что производные порядков не меньше двух определены таким образом.

Нам понадобятся еще два обозначения. Через $\mathcal{X}$ обозначим класс бочечных хаусдорфовых ЛВП таких, что существуют последовательности $\left\{f_{n}\right\},\left\{g_{n}\right\} \subset X^{\prime}$, удовлетворяюшие условиям:

(i) для любой последовательности $\left\{c_{n}\right\}$ положительных чисел существует элемент $x \in X$, для которого $g_{n}(x)=0$ при всех $n \in \mathbb{N}$ и

$$
\varlimsup_{n \rightarrow \infty} \frac{\left|f_{n}(x)\right|}{c_{n}}=+\infty
$$

(ii) для всякого ограниченного множества $B \subset E$ существует натуральное число $n=n(B)$, для которого $g_{k}(x)=0$ при $k>n$ и $x \in B$;

(iii) сушествуют элементы $x_{m} \in X$ такие, что $g_{n}\left(x_{m}\right)=\delta_{n, m}$ при всех $m, n \in \mathbb{N}$.

Через $\mathcal{Y}_{0}$ обозначим класс таких ЛВП $X$, что для всякой сходящейся к нулю последовательности $\left\{x_{n}\right\} \subset X$ существует банахов диск ${ }^{2} B$ такой, что $p\left(x_{n}\right) \rightarrow 0$, где $p$ - функционал Минковского множества $B$.

Через $\mathcal{Y}$ обозначим класс таких бочечных ЛВП $X$, что:

(a) существует банахов диск $D \subset X$ с плотной линейной оболочкой;

(б) $X$ является строгим проективным пределом ${ }^{3}$ последовательности секвенциальных ${ }^{4}$ ЛВП $X_{n} \in \mathcal{Y}_{0}$.

\section{§3. Разрывные комплексно дифференцируемые функции}

Пусть $X \in \mathcal{X}$. Тогда существуют последовательности $\left\{f_{n}\right\},\left\{g_{n}\right\} \subset X^{\prime}$, удовлетворяюшие условиям (i)-(iii) из определения класса $\mathcal{X}$. Рассмотрим отображение $P: X \rightarrow \mathbb{K}$

$$
P(x)=\sum_{n=0}^{\infty} f_{n}(x) g_{n}(x) .
$$

В силу условия (ii) в ряде из формулы (4) для каждого $x$ лиш конечное число слагаемых отлично от нуля.

ТеОРема 1. Функиия $P$, определяемая равенством (4), всюду разрывна, всюду дифферениирума по Фреше, и производная $P^{\prime}: X \rightarrow X^{\prime}$ является непрерывным линейным оператором.

\footnotetext{
${ }^{2}$ Множество $D$ называется банаховым диском, если оно ограничено, замкнуто, выпукло, уравновешено, причем его линейная оболочка $X_{D}$ является банаховым пространством при наделении ее нормой $p_{D}$, где $p_{D}-$ функционал Минковского множества $D$.

${ }^{3}$ То есть существуют непрерывные открытые линейные операторы $\pi_{n}: X \rightarrow X_{n}, \rho_{n}$ : $X_{n+1} \rightarrow X_{n}$ такие, что $\pi_{n}=\rho_{n} \pi_{n+1}$ при всех $n$ и топология пространства $X$ является слабейшей, относительно которой все $\pi_{n}$ непрерывны.

${ }^{4}$ Топологическое пространство называется секвенциальным, если в нем любое секвенциально замкнутое множество замкнуто.
} 
ЗАмечаниЕ 1 . Из теоремы 1 вытекает, что функция $P$ бесконечно дифференцируема по Фреше, отображение $P^{\prime \prime}: X \rightarrow L\left(X, X^{\prime}\right)$ постоянно и $P^{(k)} \equiv 0$ при $k \geqslant 3$. В частности, $P^{(k)}$ непрерывны при всех $k \geqslant 1$.

СлЕДСТВИЕ 1. На всяком (вещественном или комплексном) ЛВП $X \in \mathcal{X}$ существует всюду разрывная бесконечно дифференцируемая по Фреше функция, производные которой непрерывны.

ДоКАЗАТЕЛЬСТВО ТЕОРЕМЫ 1. Вычисляя предел (2) для отображения (4), получаем

$$
\lim _{\substack{t \rightarrow 0 \\ t \in \mathbb{K}}} \frac{P(x+t h)-P(x)}{t}=\sum_{n=1}^{\infty}\left(f_{n}(x) g_{n}(h)+f_{n}(h) g_{n}(x)\right)
$$

для всех $x, h \in X$. Для любого $x \in X$ отображение

$$
T x(h)=\sum_{n=1}^{\infty}\left(f_{n}(x) g_{n}(h)+f_{n}(h) g_{n}(x)\right)
$$

является линейным функционалом на $X$. Непрерывность функционала $T x$ вытекает из бочечности $X$ и того, что $T x$ является поточечным пределом последовательности непрерывных линейных функционалов

$$
T_{n} x(h)=\sum_{k=1}^{n}\left(f_{k}(x) g_{k}(h)+f_{k}(h) g_{k}(x)\right) .
$$

Следовательно, формула (6) определяет линейный оператор $T: X \rightarrow X^{\prime}$. В силу условия (ii) последовательность $T_{n} x$ сходится к $T x$ в сильной топологии пространства $X^{\prime}$ для всякого $x \in X$. Стало быть, оператор $T$ непрерывен, так как $X$ бочечно и $T$ является поточечным пределом последовательности непрерывных линейных операторов $T_{n}$. Таким образом, функция $P$ дифференцируема по Гато, и отображение $P^{\prime}: X \rightarrow X^{\prime}$ является непрерывным линейным оператором.

Покажем теперь, что $P$ всюду дифференцируемо по Фреше. Пусть $x \in X$ и $B \subset X$-ограниченное множество. В силу условия (ii) существует $n=n(B, x) \in \mathbb{N}$, для которого $g_{k}(h)=0$ при всех $h \in B \cup\{x\}$. Тогда для всякого $h \in B$ левая часть равенства (5) совпадает с величиной

$$
\lim _{t \rightarrow 0} \frac{P_{n}(x+t h)-P_{n}(x)}{t}, \quad P_{n}(x)=\sum_{k=0}^{n} f_{k}(x) g_{k}(x) .
$$

Предел в формуле (8), очевидно, равномерен по $h \in B$; поэтому функция $P$ дифференцируема по Фреше.

Пусть $x \in X$. Легко видеть, что $P(x+h)=P(h)+P(x)+T x(h)$. Поскольку линейный функционал $T x$ непрерывен, имеем, что непрерывность (разрывность) $P$ в точке $x$ эквивалентна непрерывности (разрывности) $P$ в нуле. Остается проверить разрывность $P$ в нуле. Пусть $U$ - окрестность нуля в $X$. В силу условия (iii) для каждого $n \in \mathbb{N}$ можно выбрать элемент $x_{n} \in U / 2$ такой, что $g_{m}\left(x_{n}\right)=0$ при 
$m \neq n$ и $g_{n}\left(x_{n}\right) \neq 0$. Согласно условию (3) при $c_{n}=\max \left\{\left|f_{n}(x)\right|, 1 /\left|g_{n}\left(x_{n}\right)\right|\right\}$ существуют $y \in X$, для которого $g_{n}(y)=0$ при всех $n \in \mathbb{N}$, и бесконечное множество $A \subset \mathbb{N}$ такие, что

$$
\lim _{\substack{n \rightarrow \infty \\ n \in A}}\left|f_{n}(y) g_{n}\left(x_{n}\right)\right|=+\infty, \quad \lim _{\substack{n \rightarrow \infty \\ n \in A}} \frac{f_{n}\left(x_{n}\right)}{f_{n}(y)}=0 .
$$

Выберем $c>0$ такое, что $2 c y \in U$. Тогда $h_{n}=c y+x_{n} \in(U+U) / 2=U$ при всех $n \in \mathbb{N}$. Стало быть, при $n>k$ мы имеем

$$
P\left(h_{n}\right)=\sum_{m=0}^{\infty} f_{m}\left(c y+x_{n}\right) g_{m}\left(c y+x_{n}\right)=\left(c f_{n}(y)+f_{n}\left(x_{n}\right)\right) g_{n}\left(x_{n}\right) .
$$

Из формулы (9) следует, что $\varlimsup_{n \rightarrow \infty}\left|P_{n}\left(h_{n}\right)\right|=\infty$, и, значит, функция $P$ неограничена на любой окрестности нуля. Следовательно, $P$ разрывна в нуле, а значит, и всюду.

Покажем, что класс $\mathcal{X}$ содержит один из известных классов ЛВП.

ПРЕДЛОЖЕНИЕ 1. Пусть X - строгий индуктивный предел строго возрастающей последовательности $\left\{X_{n}\right\}$ ненормируемых пространств Фреше. Тогда $X \in \mathcal{X}$.

ДокаЗАТЕЛЬСТво. Можно считать, что $\operatorname{dim} X_{n+1} / X_{n} \geqslant n$ для всякого $n \in \mathbb{N}$ (если это не так, то надо от $X_{n}$ перейти к подходящей подпоследовательности). Построим по индукции последовательности $\left\{x_{n}\right\} \subset X$ и $\left\{g_{n}\right\} \subset X^{\prime}$ такие, что

$$
x_{n} \in X_{n+1} \backslash X_{n},\left.\quad g_{n}\right|_{X_{n}} \equiv 0 .
$$

Пусть $x_{1} \in X_{2} \backslash X_{1}$. Согласно теореме Хана-Банаха существует $g_{1} \in X^{\prime}$ такой, что $g_{1}\left(x_{1}\right)=1$ и $\left.g_{1}\right|_{X_{1}} \equiv 0$. Основание индукции построено. Пусть теперь $n \geqslant 2$ и $x_{j}, g_{j}$, удовлетворяюшие условию (10), уже построены. Поскольку $\operatorname{dim} X_{n+1} / X_{n} \geqslant n$, мы имеем, что существует $x_{n} \in X_{n+1} \backslash X_{n}$ такой, что $g_{1}\left(x_{n}\right)=\cdots=g_{n-1}\left(x_{n}\right)=0$. По теореме Хана-Банаха существует $g_{n} \in X^{\prime}$ такой, что $g_{n}\left(x_{n}\right)=1$ и $\left.g_{n}\right|_{X_{n}} \equiv 0$. Шаг индукции описан и тем самым последовательности $\left\{x_{n}\right\} \subset X$ и $\left\{g_{n}\right\} \subset X^{\prime}$, удовлетворяюшие условию (10), построены.

Поскольку $X_{1}$ является ненормируемым пространством Фреше, существуют [20], [21] $\varphi_{n} \in X_{1}^{\prime}$ такие, что для любой последовательности $\left\{c_{n}\right\} \in \mathbb{K}$ существует $x \in X$, для которого $\varphi_{n}(x)=c_{n}$ при всех $n \in \mathbb{N}$. По теореме Хана-Банаха сушествуют $f_{n} \in X^{\prime}$ такие, что $\left.f_{n}\right|_{X_{1}}=\varphi_{n}$ при всех $n \in \mathbb{N}$. Покажем, что последовательности $\left\{f_{n}\right\}$ и $\left\{g_{n}\right\}$ удовлетворяют условиям (i)-(iii). Пусть $c_{n}>0$. Выберем $x \in X_{1}$, для которого $f_{n}(x)=\varphi_{n}(x)=n c_{n}$ при всех $n \in \mathbb{N}$. Значит, $\left|f_{n}(x)\right| / c_{n}=n \rightarrow \infty$. Поскольку $\left.g_{k}\right|_{X_{1}} \equiv 0$, имеем $g_{k}(x)=0$ при всех $k \in \mathbb{N}$, что доказывает (i). Пусть $B$ - ограниченное множество $X$. Тогда сушествует $n \in \mathbb{N}$, для которого $B \subset X_{n}$ [7], [8]. В силу (10) $\left.g_{m}\right|_{B} \equiv 0$ при $m \geqslant n$ и условие (ii) проверено. Условие (iii) для определенной выше последовательности $\left\{x_{n}\right\}$ также вытекает из (10). 
СледСТвиЕ 2. Поскольку $\mathcal{D} \in \mathcal{X}$, то на $\mathcal{D}$ существует бесконечно $\mathbb{K}-\partial и ф-$ ференцируемая всюду разрывная функция.

ЗАмечАниЕ 2 . Для $X=\mathcal{D}$ функционалы $f_{n}$ и $g_{n}$, удовлетворяюшие условиям (i)-(iii), можно указать в явном виде. Например, $f_{n}(x)=x^{(n)}(0)$ и $g_{n}(x)=x(n)$.

\section{§4. Поточечная сходимость степенных рядов}

ПРЕДЛОЖЕНИЕ 2. Пусть $(X, p)$ - банахово пространство, $а(Y, q)$ - нормированное пространство над полем $\mathbb{K}, \varepsilon>0, a_{0} \in Y, x_{0} \in X, \varphi_{n} \in \mathcal{B}_{s}^{n}(X, Y)$, $n \in \mathbb{N}$, все $\varphi_{n}$ непрерывны и ряд

$$
a_{0}+\sum_{n=1}^{\infty} \widehat{\varphi}_{n}\left(x-x_{0}\right)
$$

(см. (1)) сходится для каждого $x \in X$ такого, что $p\left(x-x_{0}\right)<\varepsilon$. Тогда существует $\delta>0$, для которого $p_{q}^{*}\left(\varphi_{n}\right)=O\left(\delta^{n}\right)$.

ЗАмечАниЕ 3 . Если пространство $X$ конечномерно, то существует оценка сверху для $\delta$ из предложения 2 , которая зависит лиш от $\varepsilon$ и размерности $X$. K сожалению, эта оценка стремится к бесконечности с ростом размерности. Тем самым предложение 2 нельзя вывести из его конечномерного аналога.

Для доказательства предложения 2 нам потребуется следующая лемма.

Лемма 1. Пусть $X$ и $Y$ - векторные пространства над полем $\mathbb{K}, n \in \mathbb{N}$, $\varphi \in \mathcal{B}_{s}^{n}(X, Y), \quad p$ и $q$ - полунормы на $X$ и $Y$ соответственно такие, что $p_{q}^{*}(\varphi)<+\infty$. Тогда существует $y \in X$, для которого $p(y)=1$ u $q(\widehat{\varphi}(y)) \geqslant$ $p_{q}^{*}(\varphi) 6^{-n}$.

ДокАЗАТЕЛЬСТво. Возьмем $x_{1}, \ldots, x_{n} \in X$ такие, что

$$
p\left(x_{j}\right) \leqslant 1, \quad j=1, \ldots, n, \quad q\left(\varphi\left(x_{1}, \ldots, x_{n}\right)\right) \geqslant 0.9 p_{q}^{*}(\varphi) .
$$

Положим $N_{n}=\{1, \ldots, n\}$ и $\Sigma(x, A)=\sum_{j \in A} x_{j}$ для $A \subseteq N_{n}$. Через $|A|$ будем обозначать число элементов множества $A$. Нетрудно проверить, что

$$
\varphi\left(x_{1}, \ldots, x_{n}\right)=\frac{1}{n !} \sum_{A \subseteq N_{n}}(-1)^{|A|+n} \widehat{\varphi}(\Sigma(x, A)) .
$$

В силу (12) и (13) существует $A \subseteq N_{n}$ такое, что

$$
q(\widehat{\varphi}(\Sigma(x, A))) \geqslant 0.9 n ! p_{q}^{*}(\varphi) 2^{-n} .
$$

Поскольку $p(\Sigma(x, A)) \leqslant|A| \leqslant n$ при $y=\Sigma(x, A) / p(\Sigma(x, A))$, мы имеем

$$
q(\widehat{\varphi}(y)) \geqslant \frac{9 n ! p_{q}^{*}(\varphi)}{10 \cdot(2 n)^{n}} \geqslant \frac{9(n / e)^{n} p_{q}^{*}(\varphi)}{10 \cdot(2 n)^{n}}=\frac{9 p_{q}^{*}(\varphi)}{10 \cdot(2 e)^{n}} \geqslant \frac{p_{q}^{*}(\varphi)}{6^{n}} .
$$


ДОКАЗАТЕЛЬСТВО ПРЕДЛОЖЕНИЯ 2. БеЗ ограничения Общности МожНо считать, что $x_{0}=0, a_{0}=0, \mathbb{K}=\mathbb{R}$ и $\varepsilon=1$. Предположим, что искомое $\delta$ не существует. Тогда

$$
\varlimsup_{n \rightarrow \infty}\left(p_{q}^{*}\left(\varphi_{n}\right)\right)^{1 / n}=+\infty .
$$

Пусть $n_{0}=0$ и $x_{0}=0$. Построим по индукции последовательности $\left\{n_{k}\right\} \subset \mathbb{N}$ и $\left\{x_{k}\right\} \subset X$ такие, что

$$
\begin{gathered}
n_{k}>n_{k-1} \quad \forall k \in \mathbb{N}, \\
p\left(x_{k}\right)<1 / 2 \quad \forall k \in \mathbb{N}, \\
p\left(x_{k}-x_{k-1}\right)<2^{-k} \quad \forall k \in \mathbb{N}, \\
q\left(\widehat{\varphi}_{n_{j}}\left(x_{k}\right)\right)>1 \quad \forall k \in \mathbb{N}, \quad j=1, \ldots, k .
\end{gathered}
$$

В силу леммы 1 и формулы (14) сушествуют $x_{1} \in X$ и $n_{1} \in \mathbb{N}$ такие, что $p\left(x_{1}\right)<1 / 2$ и $q\left(\widehat{\varphi}_{n_{1}}\left(x_{1}\right)\right)>1$. Основание индукции построено. Пусть $m \in \mathbb{N}$, $m \geqslant 2$, и $n_{k}, x_{k}$ при $k<m$, удовлетворяюшие условиям (15)-(18), уже построены. Поскольку отображение $\widehat{\varphi}_{l}$ непрерывно, сушествует $\nu \in\left(0,2^{-m}\right)$ такое, что

$$
\begin{gathered}
p(x)<\frac{1}{2}, \quad q\left(\widehat{\varphi}_{n_{j}}(x)\right)>1, \quad j=1,2, \ldots, m-1, \\
x \in X, \quad p\left(x-x_{m-1}\right)<\nu .
\end{gathered}
$$

В силу леммы 1 и формулы (14) существуют $y \in X$ и $n_{m} \in \mathbb{N}$ такие, что $n_{m}>$ $n_{m-1}, p(y)<\nu$ и $q\left(\widehat{\varphi}_{n_{m}}(y)\right)>2^{n_{m}}$. По теореме Хана-Банаха сушествует $\psi \in Y^{\prime}$, для которого

$$
\|\psi\|=\sup \{|\psi(y)|: q(y) \leqslant 1\}=\frac{1}{q\left(\widehat{\varphi}_{n_{m}}(y)\right)}, \quad \psi\left(\widehat{\varphi}_{n_{m}}(y)\right)=1 .
$$

Пусть $\mathcal{P}$ - множество всех полиномов вида

$$
h(s)=s^{n_{m}}+\sum_{l=0}^{n_{m}-1} \alpha_{l} s^{l}, \quad \alpha_{l} \in \mathbb{R} .
$$

Очевидно, полином $r(s)=\psi\left(\widehat{\varphi}_{n_{m}}\left(x_{m-1}+s y\right)\right)$ принадлежит $\mathcal{P}$. Значит,

$$
\sup _{s \in[-1,1]}|r(s)| \geqslant \inf _{h \in \mathcal{P}} \sup _{s \in[-1,1]}|h(s)| .
$$

Величина $\sup _{s \in[-1,1]}|h(s)|$ в формуле (20) минимальна (и при этом равна $2^{1-n_{m}}$ ) для полинома $q=2^{1-n_{m}} T_{n_{m}}(s)$, где $T_{k}(t)=\cos (k \arccos t)$ - полиномы Чебышева [13]. Значит, в силу (20) сушествует $s \in[-1,1]$, для которого выполнено неравенство $\psi\left(\widehat{\varphi}_{n_{m}}\left(x_{m-1}+s y\right)\right) \geqslant 2^{1-n_{m}}$. Пусть $x_{m}=x_{m-1}+s y$. Тогда

$$
q\left(\widehat{\varphi}_{n_{m}}\left(x_{m}\right)\right) \geqslant \frac{2^{1-n_{m}}}{\|\psi\|}=2^{1-n_{m}} q\left(\widehat{\varphi}_{n_{m}}(y)\right)>1
$$


Вьполнение всех прочих условий из (15)-(18) для $x_{m}$ вытекает из (19) и неравенств $p(y)<\nu<2^{-m}$. Последовательности $\left\{x_{m}\right\}$ и $\left\{n_{m}\right\}$ построены.

В силу соотношения (17) $\left\{x_{k}\right\}$ является последовательностью Коши в $X$. Следовательно, предел $w=\lim _{k \rightarrow \infty} x_{k} \in X$ существует. Переходя к пределу в (18), получаем, что $q\left(\widehat{\varphi}_{n_{j}}(w)\right) \geqslant 1$ для всякого $j \in \mathbb{N}$. Значит, ряд (11) расходится для $x=w$. С другой стороны, условие $(16)$ влечет неравенство $p(w) \leqslant 1 / 2$. Поскольку $\varepsilon=1$, ряд (11) сходится при $x=w$. Полученное противоречие завершает доказательство.

ЗАмЕчАниЕ 4 . Из предложения 2 следует, что степенной ряд со значениями в нормированном пространстве, поточечно сходяшийся в некотором шаре банахова пространства, сходится равномерно в меньшем шаре. Отсюда, в свою очередь, вытекает, что функция со значениями в нормированном пространстве, определенная степенным рядом, поточечно сходящимся в некотором шаре банахова пространства, аналитична в этом шаре в обычном смысле [11], [12].

\section{§5. Непрерывность комплексно дифференцируемых по Гато функций}

Пусть $X$ - комплексное ЛВП и $U$ - открытое множество $X$. Согласно классической теореме об аналитичности голоморфной функции [17] для всякой комплексно дифференцируемой по Гато функции $f: U \rightarrow \mathbb{C}$ и любого $x \in U$ существуют окрестность $V_{x}$ точки $x$ и последовательность $\left\{\varphi_{n}^{x}\right\} \subset \mathcal{B}_{s}^{n}(X)$ (по определению дифференцируемости по Гато линейньй функционал $\varphi_{1}^{x}=f^{\prime}(x)$ непрерывен, но остальные $\varphi_{n}^{x}$ могут быть, вообше говоря, разрьвными) такие, что ряд

$$
f(x)+\sum_{n=1}^{\infty} \widehat{\varphi}_{n}^{x}(y-x)
$$

сходится к $f(y)$ для всякого $y \in V_{x}$. Отметим, что функционалы $\varphi_{n}^{x}$ из (21) однозначно определяются по $f$ и $x$, а в качестве $V_{x}$ можно взять любое открытое множество такое, что $V_{x} \subset U$ и множество $V_{x}-x$ выпукло и уравновешено.

Если $x \in U, h \in X$ и $y \in V_{x}$, то, поскольку сходяшийся ряд Тейлора функции двух комплексных переменных можно почленно дифференцировать (мы говорим здесь о сужении функции $f$ и ряда (21) на пересечение $V_{x}$ c аффинным подпространством, натянутым на векторы $x, y$ и $h)$, мы имеем

$$
f^{\prime}(y)(h)=D_{h} f(y)=\sum_{n=1}^{\infty} n \varphi_{n}^{x}(h, y-x, \ldots, y-x) .
$$

ЛЕмма 2. Пусть $X$ - комплексное бочечное пространство, $U$-открытое подмножество $X$ и $f: U \rightarrow \mathbb{C}$ - комплексно дифференцируемая по Гато функиия. Тогда функционалы $\varphi_{n}^{x}$ из формуль (21) раздельно непрерывны.

ДокАЗАТЕЛЬСТво. Без ограничения общности можно считать, что $0 \in U$, $x=0$ и $f(0)=0$. Очевидно, $f^{\prime}(0)=\varphi_{1}^{0}$, и, значит, линейный функционал $\varphi_{1}^{0}$ непрерывен. Мы докажем раздельную непрерывность $\varphi_{n}^{0}$ для $n=2,3, \ldots$ по индукции. Пусть $n \geqslant 2$ и функционалы $\varphi_{1}^{0}, \ldots, \varphi_{n-1}^{0}$ раздельно непрерывны. Пусть $y \in U$ 
и $h \in X$. В силу формулы (22), предположения индукции и дифференцируемости $f$ по Гато мы имеем, что линейное отображение

$$
\psi_{y}(h)=f^{\prime}(y)(h)-\sum_{k=1}^{n-1} k \varphi_{k}^{0}(h, y, \ldots, y)=\sum_{k=n}^{\infty} k \varphi_{k}^{0}(h, y, \ldots, y)
$$

непрерывно по $h$. Поскольку поточечный предел последовательности непрерывных линейных операторов, определенных на бочечном ЛВП, является непрерывным линейным оператором, мы получаем, что функционал

$$
\eta_{y}(h)=\varphi_{n}^{0}(y, \ldots, y, h)=\lim _{t \rightarrow 0} t^{-n} \psi_{t y}(h)
$$

непрерывен для всякого $y \in X$. Поскольку

$$
\varphi_{n}^{0}\left(h, u_{1}, \ldots, u_{n-1}\right)=\sum_{A \subseteq N_{n-1}}(-1)^{n-1-|A|} \eta_{\Sigma(u, A)}(h)
$$

где $\Sigma(u, A)=\sum_{j \in A} u_{j}$, мы имеем, что функционалы $h \mapsto \varphi_{n}^{0}\left(u_{1}, \ldots, u_{n-1}, h\right)$ также являются непрерывными. Так как полилинейный функционал $\varphi_{n}^{0}$ симметричен и непрерывен по последней переменной, мы получаем, что $\varphi_{n}^{0}$ раздельно непрерывен.

СлЕДСТвИЕ 3. Пусть $U$ - открытое подмножество комплексного банахова пространства $X$ u $f: U \rightarrow \mathbb{C}$ - комплексно дифференцируемая по Гато функиия. Тогда функиия $f$ бесконечно дифференцируема по Фреше. В частности, $f$ и все ее производные $f^{(n)}$ непрерывны.

ДокАЗАТЕЛЬСТВО. Пусть $x \in U$. По лемме 2 полилинейные функционалы $\varphi_{n}^{x}$ из формулы (21) раздельно непрерывны для всякого $n \in \mathbb{N}$. По теореме Банаха-Штейнгауза [7], [8] $\varphi_{n}^{x}$ непрерывны. В силу предложения 2 сушествует выпуклая уравновешенная окрестность нуля $B$ в $X$ такая, что ряд (21) равномерно сходится на множестве $x+B$. Аналогичное утверждение справедливо для рядов из $n$-х производных членов ряда (21). Следовательно, функция $f$ бесконечно дифференцируема по Гато, непрерывна и имеет непрерывные производные всех порядков, что влечет её бесконечную дифференцируемость по Фреше [6].

Теорема 2. Пусть $\mathbb{K}=\mathbb{C}, \quad X \in \mathcal{Y}, U$ - открытое подмножество $X$ и функция $f: U \rightarrow \mathbb{C}$ комплексно дифференцируема по Гато. Тогда $f$ непрерывна.

ДоКАЗАТЕЛЬСТВО. Предположим, что $f$ разрьвна. Не ограничивая обшности рассуждений, можно считать, что $f$ разрывна в точке $0 \in U$, множество $U$ выпукло и уравновешено и $f(0)=0, f^{\prime}(0)=0$. Поскольку $U$ выпукло и уравновешено, ряды (21) и (22) сходятся для всех $y \in U$. В силу условия (а) сушествует банахов диск $D \subset X$ с линейной оболочкой $X_{D}$, плотной в $X$. Согласно условию (б) $X$ является строгим проективным пределом последовательности $\left\{X_{n}\right\}$ секвенциальных ЛВП 
относительно отображений $\pi_{n}: X \rightarrow X_{n}, \rho_{n}: X_{n+1} \rightarrow X_{n}$. Нам потребуются также сопряженные операторы $\pi_{n}^{\prime}: X_{n}^{\prime} \rightarrow X^{\prime}$. Пусть

$$
A_{n}=\left\{u \in X_{D} \cap U: f^{\prime}(u) \in \pi_{n}^{\prime}\left(X_{n}^{\prime}\right)\right\} .
$$

Поскольку $X_{D} \cap U=\bigcup_{n=1}^{\infty} A_{n}$, в силу теоремы Бэра [19] существуют непустое открытое в банаховом пространстве $X_{D}$ множество $W \subset X_{D} \cap U$ и $n \in \mathbb{N}$ такие, что $A_{n} \cap W$ плотно в $W$ в топологии $X_{D}$. Так как топология $X_{D}$ сильнее индуцированной из $X$, сужение $f_{1}=\left.f\right|_{X_{D} \cap U}: X_{D} \cap U \rightarrow \mathbb{C}$ является комплексно дифференцируемым по Гато. Положим $Y_{n}=\operatorname{ker} \pi_{n}$. Тогда для всех $y \in Y_{n}$ и $u \in A_{n} \cap W$ имеем $D_{y} f(u)=f_{1}^{\prime}(u)(y)=0$. По следствию 3 отображение $f_{1}^{\prime}$ непрерывно. Поскольку множество $A_{n} \cap W$ плотно в $W$, то $f_{1}^{\prime}(u)(y)=D_{y} f(u)=0$ для всех $y \in Y_{n}$ и $u \in W$. Из обычной теоремы единственности, примененной ко всем сужениям $f_{1}^{\prime}(\cdot)(y)$ на пересечения одномерных аффинных подпространств $X_{D}$ с множеством $W$, вытекает, что $f_{1}^{\prime}(u)(y)=D_{y} f(u)=0$ для всех $y \in Y_{n}$ и $u \in U \cap X_{D}$. В силу (22) получаем

$$
\sum_{n=2}^{\infty} n \varphi_{n}^{0}(y, u, \ldots, u)=0
$$

для любых $y \in Y_{n}$ и $u \in U \cap X_{D}$.

Рассматривая равенство (24) для $u$ из одномерных аффинных подпространств $X_{D}$ и снова применяя теорему единственности, имеем, что $\varphi_{n}^{0}(y, u, \ldots, u)=0$ для всех $n \in \mathbb{N}, y \in Y_{n}$ и $u \in X_{D}$. В силу формулы (23) получаем, что $\varphi_{n}^{0}\left(y, u_{1}, \ldots, u_{n-1}\right)=0$ для всех $n \geqslant 2, y \in Y_{n}$ и $u_{1}, \ldots, u_{n-1} \in X_{D}$. Поскольку $X$ бочечно, из леммы 2 вытекает раздельная непрерывность $\varphi_{n}^{0}$. Так как $X_{D}$ плотно в $X$, то $\varphi_{n}^{0}\left(y, u_{1}, \ldots, u_{n-1}\right)=0$ для всех $n \geqslant 2, y \in Y_{n}$ и $u_{1}, \ldots, u_{n-1} \in X^{\prime}$. В силу соотношения (22) $D_{y} f(u)=0$ для всех $y \in Y_{n}$ и $u \in U$. Стало быть,

$$
f(u)=f(u+y), \quad u, u+y \in U, \quad y \in Y_{n} .
$$

Формула (25) означает, что $g \circ T=f$ для некоторого отображения $g: V \rightarrow \mathbb{C}$, где $V=\pi_{n}(U) \subset X_{n}$. Легко видеть, что $g$ дифференцируема по Гато и разрывна в нуле (иначе $f$ непрерывна в нуле как композиция непрерывных отображений $\pi_{n}$ и $\left.g\right)$. Так как пространство $X_{n}\left(\in \mathcal{Y}_{0}\right)$ секвенциально, существуют $x_{k} \in X_{n}$, $k \in \mathbb{N}$, для которых $x_{k} \rightarrow 0$ и $g\left(x_{k}\right) \not \rightarrow 0$. В силу определения $\mathcal{Y}_{0}$ сушествует банахов диск $B \subset X_{n}$ такой, то $p\left(x_{k}\right) \rightarrow 0$, где $p$ - функционал Минковского множества $B$. Поскольку топология банахова пространства $Z=\left(X_{n}\right)_{B}$ сильнее топологии, индуцированной из $X$, сужение $\left.g\right|_{Z \cap U}: Z \cap U \rightarrow \mathbb{C}$ комплексно дифференцируемо по Гато. В силу следствия 3 это сужение непрерывно, что противоречит соотношениям $p\left(x_{n}\right) \rightarrow 0$ и $g\left(x_{n}\right) \not \rightarrow 0$.

Следуюшее предложение показывает, что класс $\mathcal{Y}$ также содержит один из известных классов ЛВП.

ПРЕДЛОЖЕНИЕ 3 . Пусть $X-$ строгий проективный предел последовательности $X_{n}$ пространств класса $\mathcal{D} \mathcal{F} \mathcal{S}$. Тогда $X \in \mathcal{Y}$. 
ДокАЗАТЕЛьСтво. Поскольку всякое пространство класса $\mathcal{D F} \mathcal{F}$ является пространством Шварца-Монтеля [15], [16] (т.е. всякое ограниченное множество является относительно компактным в некотором банаховом пространстве, порожденном компактным диском), мы имеем, что всякое пространство класса $\mathcal{D} \mathcal{F} \mathcal{S}$ принадлежит $\mathcal{Y}_{0}$ и является секвенциальным, что гарантирует выполнение условия (б) для $X$. Бочечность $X$ вытекает из бочечности пространств класса $\mathcal{D} \mathcal{F} \mathcal{S}$ и сохранении бочечности при строгих проективных пределах. В каждом $X_{n}$ (как и в любом пространстве класса $\mathcal{D} \mathcal{F} \mathcal{S}$ ) можно выбрать компактный диск $K_{n}$ с плотной линейной оболочкой. Нетрудно проверить, что существует последовательность положительных чисел $c_{n}$, для которой множество $M=\bigcup_{n=1}^{\infty} c_{n} K_{n}$ компактно в $X$. Замыкание $D$ выпуклой уравновешенной оболочки множества $M$ будет компактным, а значит, банаховым диском [21] в $X$ с плотной линейной оболочкой, что доказывает выполнение условия (а).

СЛЕДСТВИЕ 4. Комплексное пространство $\mathcal{D}^{\prime}$ принадлежит $\mathcal{Y}$, и, значит, всякая комплексно дифферениируемая по Гато функиия на $\mathcal{D}^{\prime}$ непрерывна.

ДокАЗАТЕЛЬСтво. Достаточно применить предложение 3 и теорему 2, поскольку $\mathcal{D}^{\prime}$ является строгим проективным пределом последовательности пространств $\mathcal{D}_{n}^{\prime}$, являющихся сильными сопряженными к ядерным пространствам Фреше [15], [18] (тем самым $\left.\mathcal{D}_{n}^{\prime} \in \mathcal{D} \mathcal{F} \mathcal{S}\right)$.

Следует отметить, что пространства класса $\mathcal{Y}$ не обязаны быть секвенщиальными. Например, $\mathcal{D}^{\prime}$ не является секвенциальным [2]. Отметим в заключение, что остается открытой проблема характеризации в независимых терминах класса комплексных ЛВП, на которых всякая определенная на открытом подмножестве дифференцируемая по Гато функция непрерьвна.

\section{Список литературы}

1. Авербух В.И., Смолянов О.Г. Различные определения дифференцируемости в линейных топологических пространствах // УМН. 1968. Т. 23. №4. С. 67-116.

2. Smolyanov O. G. Non-closed sequentially closed subsets of locally convex spaces and applications // Note di Math. 1992. V. 12. P. 237-244.

3. Шкарин C. A. Точки непрерывности дифференцируемых по Гато отображений // Сиб. матем. журн. 1992. Т. 33. С. 905-913.

4. Смолянова М. О. Бесконечно дифференцируемые разрывные функции на пространстве $\mathcal{D}^{\prime}$ // Вестн. МГУ. Сер. 1. 1993. № 4. С. 46-48.

5. Смолянова М. О. Непрерывно дифференцируемая разрывная функция на пространстве $\mathcal{D} / /$ Изв. РАН. Сер. матем. 1995. Т. 59. № 5. С. 1077-1082.

6. Смолянов О.Г. Анализ на топологических векторных пространствах и его приложения. М.: Изд-во МГУ, 1979.

7. Робертсон A., Робертсон В. Топологические векторные пространства. М.: Мир, 1967.

8. Шефер X. Топологические векторные пространства. М.: Мир, 1971.

9. Энгелькинг Р. Общая топология. М.: Мир, 1986.

10. Bonet J., Carreras P. Barrelled locally convex spaces. Amsterdam: North-Holland, 1987.

11. Mujica J. Complex analysis in Banach spaces. Amsterdam: North-Holland, 1986.

12. Dineen $S$. Complex analysis of infinite dimensional spaces. London: Springer, 1999.

13. Алексеев B. М., Тихомиров В. М., Фомин С. В. Оптимальное управление. М.: Наука, 1979. 
14. Köthe G. Topological vector spaces. I. N.Y.: Springer, 1969.

15. Köthe G. Topological vector spaces. II. N.Y.: Springer, 1979.

16. Grothendieck A. Sur les espaces (F) et (DF) // Summa Brasil Math. 1954. V. 3. P. 57-123.

17. Титчмари E. Теория функций. М.: Наука, 1980.

18. Vogt $D$. Sequence space representations of the spaces of test functions and distributions // Lectures in pure and applied mathematics / Ed. G.I. Zapata. V. 83. N.Y.: Marcel Dekker, 1983.

19. Колмогоров A.H., Фомин C. В. Элементы теории функций и функционального анализа. М.: Наука, 1989.

20. Eidelheit. M. Zur Theorie der System linearer Gleichungen // Studia Math. 1936. V. 6. P. 139-148.

21. Bonet J., Carreras P. Barrelled locally convex spaces // North-Holland Mathematics Studies. V. 131. Amsterdam: North-Holland, 1987.

МГУ им. М. В. Ломоносова

Поступило в редакцию

E-mail : smolyanov@yandex.ru

14.11.2003

sshkarin@hotmail.com 